\title{
Effect of Curcumin on Healing of Extraction Sockets in Type II Diabetic Patients- A Pilot Study
}

\author{
R. Mugilan ${ }^{1}$, Riddhika Jayaswal ${ }^{2}$, Sowmya R. ${ }^{3}$, Sunil Sunny Vincent ${ }^{4}$, Vaishali K. ${ }^{5}$, Kavitha Prasad ${ }^{6}$
}

\begin{abstract}
${ }^{1}$ Faculty of Dental Sciences, M.S. Ramaiah University of Applied Sciences, Bengaluru, Karnataka, India. ${ }^{2}$ Faculty of Dental Sciences, M.S. Ramaiah University of Applied Sciences, Bengaluru, Karnataka, India. ${ }^{3}$ Faculty of Dental Sciences, M.S. Ramaiah University of Applied Sciences, Bengaluru, Karnataka, India. ${ }^{4}$ Faculty of Dental Sciences, M.S. Ramaiah University of Applied Sciences, Bengaluru, Karnataka, India. ${ }^{5}$ Department of Prosthodontics, M.S.

Ramaiah University of Applied Sciences, Bengaluru, Karnataka, India. ${ }^{6}$ Department of Oral and Maxillofacial Surgery, M.S. Ramaiah University of Applied Sciences, Bengaluru, Karnataka, India.
\end{abstract}

\section{ABSTRACT}

\section{BACKGROUND}

Curcuma longa, commonly called Turmeric, is a plant, belonging to family Zingiberaceae (ginger family) which has been used in traditional medicine for the management of several diseases such as diabetic ulcers, rheumatic conditions, external wounds and cough. Diferuloylmethane is the most active chemical extract found in Curcumin, has been shown to exert antioxidant, antiaging, antiinflammatory, immunomodulatory, wound healing, antitumoral and antipsoriatic effects. Delayed wound healing is a very common complication associated with diabetic patients, following extractions. Recent research has suggested that curcumin has great potential to address this.

\section{METHODS}

A total of 11 patients, who satisfied the inclusion criteria (RBS $=<200 \mathrm{mg} / \mathrm{dL}$ ) were selected from the out-patient department of Oral and Maxillofacial Surgery, FDSRUAS. They were then divided into two groups - test and control, comprising of 6 in test group 5 in control group. Following the extraction, in the test group patients, Curcuma oral gel was placed, within the extraction socket. Both the groups were given standard post-extraction instructions. Healing of both study and control group extraction sockets were assessed on day 0 , day 3 and day 7 respectively.

\section{RESULTS}

The study group patients showed statistically significant reduction in the width of extraction socket on day 3 [mesial ( $p$-value $=0.03$ )] and day 7 [mesial ( $p$-value $=0.002)]$. Central (p-value $=0.03$ ) and Distal ( $\mathrm{p}$-value $=0.03)$ ) and significant reduction of pain by day $7(0.002)$.

\section{CONCLUSIONS}

Curcumin is a safe, natural herb, giving good results in the healing of the extraction socket in diabetic patients, with definite improvement in the soft tissue healing.

\section{KEY WORDS}

Curcumin, Wound Healing, Diabetes Mellitus, Extraction Socket Healing
Corresponding Author: Dr. Vaishali $K$., \#2301, Anriya Dwellington Apartment, \#1, $1^{\text {st }}$ Main, Dollars Colony, RMV $2^{\text {nd }}$ Stage, Bangalore-560094, Karnataka, India. E-mail: vaishalivijay10@gmail.com

DOI: $10.14260 /$ jemds/2020/225

Financial or Other Competing Interests: None.

How to Cite This Article:

Mugilan R, Jayaswal $R$, Sowmya $R$, et al. Effect of curcumin on healing of extraction sockets in type ii diabetic patients- a pilot study. J. Evolution Med. Dent. Sci. 2020;9(13):1045-1049, DOI: $10.14260 /$ jemds $/ 2020 / 225$

Submission 23-09-2019,

Peer Review 07-03-2020,

Acceptance 13-03-2020,

Published 30-03-2020.

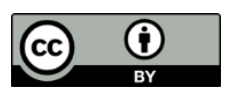




\section{BACKGROUND}

The healing of extraction sockets has always been of significant concern to a dentist for the purpose of facilitating early rehabilitation. However, in certain cases, there can be delayed wound healing or unwarranted complications post extraction, which cause a constrain both on the efforts of the Dentist to offer successful rehabilitation, and the willingness of the patient to accept dental treatment. Diabetes mellitus is a common metabolic disorder among the elderly, characterized by an inability to regulate blood glucose due to insulin resistance, thereby leading to elevated levels of Blood Glucose. Diabetes mellitus, or Type-2 Diabetes, is such a condition wherein there are increased chances of occurrence of a significant delay in wound healing or post-extraction complications, in comparison to an otherwise healthy individual.1,2,3,4,5,6 Essentially, healing of the socket post extraction occurs as a sequence of events; the formation of a blot clot immediately after the extraction, the epithelialization of the wound and subsequent formation of granulation tissue, replacement of the granulation tissue into connective tissue through the laying down of collagen by the fibroblasts, formation of woven bone over scaffold formed, and finally maturation of the woven bone into lamellar bone. ${ }^{7,8}$

These phases are mediated by the release of various cytokines and growth factors, which initially trigger local inflammation, and later Angiogenesis. A balance and smooth transition between the phases of wound healing ensures minimum complications. The reason for delayed wound healing in diabetic patients has been attributed to various factors such as leukocytes dysfunction, thickening of the basement membrane of the capillaries which results in microcirculatory deficiencies and altered permeability with the poor migration of leucocytes, Poor tissue nutrition and waste products removed from the wound site. A number of medicaments and substances have been utilised to achieve uncomplicated healing of extraction sockets, thereby hastening the process of rehabilitating the patient.

Curcuma longa, commonly called Turmeric, is a plant, belonging to family Zingiberaceae which has been used in traditional medicine for the management of several diseases such as diabetic ulcers, rheumatic conditions, external wounds and cough. Curcumin, chemically diferuloylmethane, the most active form among the extracts of the plant, has been shown to exert antioxidant, antiaging, anti-inflammatory, immunomodulatory, wound healing, antitumoral, and antipsoriatic effects. ${ }^{9}, 10,11,12$ Wound healing, the antiinflammatory, anti-infectious, apoptotic and anti-oxidant properties of Curcumin have been considered the pathways through which the compound mediates its effect.11,12 Since Turmeric is a part of his everyday diet in this part of the continent, the application of this substance, the into the extraction sockets, would very much be met with little resistance from the patient. Moreover, since the paste of turmeric has been used for dressing of wounds from time immemorial, one can always expect the patient to wilfully accept the proposition of placement of a Curcumin medicament.

In essence, by combining the aforementioned information, it can be seen that this study aims to evaluate the effectiveness of a natural-derived ingredient, curcumin, in the healing of extraction sockets in Diabetic patients.

\section{METHODS}

This randomised double blinded pilot study was conducted in the Department of Oral and Maxillofacial Surgery (OMFS), Faculty of Dental Sciences, Ramaiah University of Applied Sciences for a period of 3 months from January to March 2019. A total of 11 patients who visited the Out-patient department were selected as per the inclusion and exclusion criteria.

\section{Inclusion Criteria}

1. Patients with General random blood sugar (GRBS) $<200$ $\mathrm{mg} / \mathrm{dL}$.

2. Presenting with teeth indicated for atraumatic extraction as a result of dental caries or periodontitis.

3. Between the age of 35 to 55 .

The exclusion criteria included factors that were known to impair wound healing of the extraction socket. $3,4,5,6,9,10$

\section{Exclusion Criteria}

1. Patients with periapical infections or lesions associated with the teeth to be extracted.

2. Opposing traumatic occlusion or impinging teeth.

3. Patients habituated to the use of tobacco and/or alcohol.

4. Female patients on oral contraceptives.

5. Systemic steroids treatment.

6. Type 1 diabetes.

7. Any other uncontrolled systemic conditions.

\section{Extraction Procedure}

Written informed consent as obtained from the study subjects as per the Helsinki Protocol. The subjects were selected based on Simple Random sampling based on a table of random numbers. The study subjects were divided into two groups Study Group with 6 subjects and Control group with 5 subjects respectively. GRBS level was recorded prior to the procedure. Atraumatic extractions were performed under 2\% Lignocaine Local anaesthetic solution (Xylocaine 2\% Adrenaline, German remedies, India) by the pre-assigned Investigators under sterile and aseptic conditions. Post-extraction the socket was compressed with mild pressure. Curenext (Abbott) containing Curcuma Oral gel (Fig. 1) was placed into the extraction socket of the study group subjects using absorbable gelatin sponge, AbGel (Gopal Krishna Labs). (Fig. 2) Subjects in both groups were given standard post-operative instructions and standard analgesic (Hifenac P -INTAS Pharmaceuticals Ltd) regimen before they were discharged. Antibiotic (Novamox- $500 \mathrm{mg}-$ Cipla Ltd) was also given as per standard protocol.

The healing criteria used in this study is a modification of healing index given by Landry, Turnbull and Howley ${ }^{13}$

- Erythema- The presence or absence of erythema was noted

- Post-operative Pain (VAS Scale)- Patients were instructed on the Visual Analog Scale ${ }^{14}$ and the readings noted.

- Width of the Extraction Socket- The width was measured in millimetres at 3 different points, namely the centre, mesial and distal region of the extraction socket was measured with a Williams graduated periodontal probe.

- Suppuration- The presence or absence of suppuration was noted. 
Observations and evaluation of wound healing was performed based on the parameters mentioned above on the day of the extraction, $3^{\text {rd }}$ and $7^{\text {th }}$ days post extraction in both the groups.

\section{Statistical Analysis}

Data obtained was analysed using Statistical Package for Social Sciences [SPSS] for Windows, Version 22.0. Armonk, NY: IBM Corp. Descriptive analysis of all the explanatory parameters was done using frequency and proportions for categorical variables, whereas in Mean and Standard Deviations for continuous variables. Independent Student $t$ Test was used to compare the mean VAS scores for pain and width of extraction socket at Mesial, Central \& Distal region at different time intervals between 2 groups. Chi Square Test was used to compare the presence of erythema and Suppuration between 2 groups at different time intervals. Repeated measures of ANOVA followed by Bonferroni's post hoc analysis was used to compare the mean VAS scores and width (in mm) of socket at various regions between different time intervals in each group. The level of significance [P-Value] was set at $\mathrm{p}<0.05$.

\section{RESULTS}

\begin{tabular}{|c|c|c|c|c|c|c|}
\hline Day & Group & $\mathbf{N}$ & Mean & SD & Mean Diff & p-Value \\
\hline \multirow{2}{*}{0} & Study & 6 & 5.33 & 0.82 & \multirow{2}{*}{-0.47} & \multirow{2}{*}{0.49} \\
\hline & Control & 5 & 5.80 & 1.30 & & \\
\hline \multirow{2}{*}{3} & Study & 6 & 2.83 & 0.75 & \multirow{2}{*}{0.03} & \multirow[b]{2}{*}{0.95} \\
\hline & Control & 5 & 2.80 & 0.84 & & \\
\hline \multirow{2}{*}{7} & Study & 6 & 1.17 & 0.41 & \multirow{2}{*}{-1.23} & \multirow{2}{*}{$0.002^{*}$} \\
\hline & Control & 5 & 2.40 & 0.55 & & \\
\hline
\end{tabular}

Table 1. Comparison of Mean VAS Scores for Pain b/w the Two Groups on Day 0, 3, 7 Using Independent Student t Test

\begin{tabular}{|c|c|c|c|c|c|c|}
\hline Variables & Group & $\mathbf{N}$ & Mean & SD & Mean Diff & p-Value \\
\hline \multirow{2}{*}{ Width-Mesial } & Study & 6 & 2.83 & 0.26 & \multirow{2}{*}{-1.27} & \multirow{2}{*}{$0.03^{*}$} \\
\hline & Control & 5 & 4.10 & 1.14 & & \\
\hline \multirow{2}{*}{ Width-Centre } & Study & 6 & 5.00 & 0.89 & \multirow{2}{*}{-1.00} & \multirow{2}{*}{0.19} \\
\hline & Control & 5 & 6.00 & 1.41 & & \\
\hline \multirow{2}{*}{ Width-Distal } & Study & 6 & 3.17 & 0.52 & \multirow{2}{*}{-0.63} & \multirow{2}{*}{0.24} \\
\hline & Control & 5 & 3.80 & 1.10 & & \\
\hline \multicolumn{7}{|c|}{$\begin{array}{c}\text { Table 2. Comparison of Mean Width of the Socket (in mm) in } \\
\text { Different Regions between the Two Groups on Day } 3 \\
\text { Using Independent Student } t \text { Test }\end{array}$} \\
\hline
\end{tabular}

\begin{tabular}{|c|c|c|c|c|c|c|}
\hline Variables & Group & $\mathbf{N}$ & Mean & SD & Mean Diff & p-Value \\
\hline \multirow{2}{*}{ Width-Mesial } & Study & 6 & 1.92 & 0.38 & \multirow{2}{*}{-1.18} & \multirow{2}{*}{$0.002^{*}$} \\
\cline { 2 - 6 } & Control & 5 & 3.10 & 0.55 & & \multirow{2}{*}{$0.03^{*}$} \\
\hline \multirow{2}{*}{ Width-Centre } & Study & 6 & 3.08 & 0.74 & \multirow{2}{*}{1.72} & \multirow{2}{*}{$0.03^{*}$} \\
\cline { 2 - 5 } & Control & 5 & 4.80 & 1.35 & \\
\hline \multirow{2}{*}{ Width-Distal } & Study & 6 & 2.08 & 0.20 & \multirow{2}{*}{-1.22} & \multicolumn{2}{|c|}{} \\
\cline { 2 - 5 } & Control & 5 & 3.30 & 1.15 & & \\
\hline
\end{tabular}

Table 3. Mean Width of the Socket (in $\mathrm{mm}$ ) in Different Regions $\mathrm{b} / \mathrm{w}$ the Two Groups on Day 7 Using Independent Student $t$ Test

\begin{tabular}{|c|c|c|c|c|c|c|c|}
\hline Group & Time & $\mathbf{N}$ & Mean & SD & p-Value ${ }^{a}$ & Sig. Diff & p-Value \\
\hline \multirow{3}{*}{ Study } & Day 0 & 6 & 4.33 & 0.52 & \multirow{3}{*}{$<0.001^{*}$} & D0 Vs D3 & $0.001^{*}$ \\
\hline & Day 3 & 6 & 2.83 & 0.26 & & D0 Vs D7 & $<0.001^{*}$ \\
\hline & Day 7 & 6 & 1.92 & 0.38 & & D3 Vs D7 & $<0.001^{*}$ \\
\hline \multirow{3}{*}{ Control } & Day 0 & 5 & 5.60 & 1.56 & \multirow{3}{*}{$0.02 *$} & D0 Vs D3 & 0.07 \\
\hline & Day 3 & 5 & 4.10 & 1.14 & & D0 Vs D7 & $0.04^{*}$ \\
\hline & Day 7 & 5 & 3.10 & 0.55 & & D3 Vs D7 & 0.14 \\
\hline \multicolumn{8}{|c|}{$\begin{array}{c}\text { Table 4. Comparison of Mean Width at Mesial Region Between } \\
\text { Different Time Intervals in Each Group Using Repeated Measures of } \\
\text { ANOVA Followed by Bonferroni's Post Hoc Test }\end{array}$} \\
\hline
\end{tabular}

Although 15 patients were selected for the study, 4 of them failed to keep the follow-up appointments, so the sample size was reduced to 11 . Of these, 6 patients belonged to the study group and 5 patients to the control group. The mean GRBS in the study group patients was $160.83 \mathrm{mg} / \mathrm{dL}$, while in the control group it was $156.20 \mathrm{mg} / \mathrm{dL}$. There was a significant reduction in pain on the 7 th day among the study group patients, when compared to that of the control group ( $p$-value $=0.002$ ) (table-1). The patients in the study group showed significant reduction in the width of the extraction socket on day 3 in the Mesial region ( $p$-value $=0.03)($ table-2) and on day 7 in the Mesial (p-value $=0.002)$ Central $(p$-value $=0.03)$ and Distal (P-value $=0.03$ ) (Table-3) region. There were no statistically significant values in the comparison of Erythema and Suppuration between the Study and Control subjects.

\begin{tabular}{|c|c|c|c|c|c|c|c|}
\hline Group & Time & $\mathbf{N}$ & Mean & SD & p-Value & Sig. Diff & p-Value ${ }^{b}$ \\
\hline \multirow{3}{*}{ Study } & Day 0 & 6 & 6.58 & 1.20 & \multirow{3}{*}{$<0.001^{*}$} & D0 Vs D3 & $0.006^{*}$ \\
\hline & Day 3 & 6 & 5.00 & 0.89 & & D0 Vs D7 & $0.001^{*}$ \\
\hline & Day 7 & 6 & 3.08 & 0.74 & & D3 Vs D7 & $0.004^{*}$ \\
\hline \multirow{3}{*}{ Control } & Day 0 & 5 & 7.50 & 1.80 & \multirow{3}{*}{$0.001^{*}$} & D0 Vs D3 & $0.008^{*}$ \\
\hline & Day 3 & 5 & 6.00 & 1.41 & & D0 Vs D7 & $0.008^{*}$ \\
\hline & Day 7 & 5 & 4.80 & 1.35 & & D3 Vs D7 & $0.03^{*}$ \\
\hline
\end{tabular}

Table 5. Comparison of Mean Width at Central Region between Different Time Intervals in Each Group Using Repeated Measures of ANOVA Followed by Bonferroni's Post Hoc Test

\begin{tabular}{|c|c|c|c|c|c|c|c|}
\hline Group & Time & $\mathbf{N}$ & Mean & SD & p-Value ${ }^{a}$ & Sig. Diff & p-Value \\
\hline \multirow{3}{*}{ Study } & Day 0 & 6 & 4.75 & 0.99 & \multirow{3}{*}{$0.001^{*}$} & D0 Vs D3 & $0.03 *$ \\
\hline & Day 3 & 6 & 3.17 & 0.52 & & D0 Vs D7 & $0.004^{*}$ \\
\hline & Day 7 & 6 & 2.08 & 0.20 & & D3 Vs D7 & $0.009^{*}$ \\
\hline \multirow{3}{*}{ Control } & Day 0 & 5 & 4.90 & 1.67 & \multirow{3}{*}{$0.02^{*}$} & D0 Vs D3 & 0.15 \\
\hline & Day 3 & 5 & 3.80 & 1.10 & & D0 Vs D7 & $0.04^{*}$ \\
\hline & Day 7 & 5 & 3.30 & 1.15 & & D3 Vs D7 & 0.10 \\
\hline
\end{tabular}

Table 6. Comparison of Mean Width at Distal Region between Different Time Intervals in Each Group Using Repeated Measures of ANOVA Followed by Bonferroni's Post Hoc Test

\section{DISCUSSION}

Type 2 Diabetes is a metabolic disorder, wherein there is a relative insulin deficiency and tissue resistance to the Insulin hormone, thereby leading to elevation of the Blood glucose levels. This hampers the wound healing following any surgical procedures. Essentially, healing of the socket post extraction occurs as a sequence of events; clot formation, epithelialization, formation of granulation tissue, replacement of the granulation tissue by connective tissue, deposition of woven bone and finally maturation of the woven bone into lamellar bone. ${ }^{15,16}$ These phases are mediated by the release of various cytokines and growth factors, which initially trigger local inflammation, and later angiogenesis. A balance and smooth transition between the phases of wound healing ensures minimum complications.

The reason for delayed wound healing in diabetic patients has been investigated by various authors. Mozzati $\mathrm{M}$ et al ${ }^{17}$ reported a delay in the healing of oral wounds in diabetic patients as a result of sluggish blood flow, low growth factors and antibodies production, depressed immunity and poor angiogenesis. Priya BM ${ }^{18}$ reported thickening of the basement membrane of the capillaries, which results in microcirculatory deficiencies and altered permeability with the poor migration of leucocytes and more chances of infections in diabetic patients. ${ }^{19}$ Poor tissue nutrition and waste products removed from the wound site due to abnormal microcirculation are other causes of poor wound healing in diabetic patients as reported by Gould L et al. ${ }^{20}$ 


\section{Post Extraction Wound Healing}

Studies also have reported delay in the healing of the post extraction socket in diabetic patients, due to weak clot and granulation tissue ${ }^{21}$ leading to alveolar destruction, incomplete wound epithelialisation reduced neutrophil Chemotaxis, reduced neutrophil and Monocyte phagocytosis, and a reduction in the oxidative burst mediated cell lysis. ${ }^{3}$ Zhang $\mathrm{S}$ et $\mathrm{al}^{2}$ reported higher post-extraction socket width and depth in diabetic patients. Although there are studies which mention that there is minimum or no change in wound healing process in controlled type 2 diabetics when compared to normal patients, still there can be no general consensus drawn to eliminate the notion that wound healing is not altered in controlled type 2 diabetic patients. ${ }^{6}$

Our study was a randomised double-blind study, where total of 11 patients in the age group 46 - 63 years and mean age of 57.64 years, were divided as 5 in the control and 6 in the test group with a mean RBS of 160.83 and 156.20 respectively. On statistical analysis there was reduction in the width of the extraction socket in test group in comparison to the control group. Statistically significant difference was seen in centre region of the extraction socket on day $3(\mathrm{p}=0.03)$ (Table-2) and at all 3 regions on the day 7 (Mesial, $p=0.002$, Centre, $p=0.03$, Distal, $p=0.03$ ) (Table-3). This indicates that curcumin helps in faster wound closure mediated through its anti-inflammatory, anti-infectious, apoptotic and antioxidant properties. Studies have shown that curcumin influences all the stages of wound healing.

\section{Inflammatory Phase}

Curcumin reduces the production of the pro-inflammatory mediators - IL- 1 , and TNF- $\alpha$ which in turn reduces inflammation by reducing the prolonged activity of Macrophages and Monocytes. Curcumin brings about reduction in synthesis of Matrix Metalloproteinase 2 and 7 and reducing inflammation- mediated matrix destruction. ${ }^{8}$

\section{Proliferative Phase}

Curcumin enhances the production of granulation tissue and neovascularisation, by virtue of increased hydroxyproline concentration, which in turn indicates increased collagen synthesis in an organised manner. ${ }^{22}$

\section{Wound Contraction Phase}

Curcumin increases aggregation of Myofibroblasts and accelerates conversion of the already existing fibroblasts into Myofibroblasts, which through Actin-protein mediated activity, leads to apposition of the edges of the wound. ${ }^{9}$

\section{Remodelling Phase}

Curcumin scavenges the ROS by enhancing the production of the anti-oxidant molecules, Superoxide Dismutase and Catalase. Further, the keratinocytes multiplying in the nascent epithelium are protected from the destructive action of Xanthine Oxidase.9,23,24

\section{Apoptosis \\ Early in the process of wound healing, curcumin has been shown to cause Apoptosis of wasted and unwanted cells at the site of the healing wound. ${ }^{8}$}

With regards to pain, our study group showed statistically significant reduction on day 7 when compared to the control group $[(\mathrm{p}=0.002)$ Table-1]. With regard to the width of the extraction socket, Within the study group at all the 3-time interval and at 3 different regions (mesial- $<0.001^{*}$, central$<0.001^{*}$ and distal $-0.001^{*}$ ) (table-4, 5) also statistical significant difference was seen. Similar finding has been reported in the study conducted by Maulina T.25 The reduction in pain can be attributed to the analgesic property of curcumin due to inhibition of the pro inflammatory cytokines such as interleukin (IL) 6, IL 8, as well as PGE2.26,27,28,29 To the common man who walks into the oral surgeon's office, Turmeric is a part of his everyday diet. The application of an everyday substance, the Curcumin compound extracted from Turmeric, into the extraction sockets, would very much be met with little resistance from the patient. Moreover, since the paste of turmeric has been used for dressing of wounds from time immemorial, one can always expect the patient to wilfully accept the proposition of placement of a Curcumin medicament. At the same time, the result of the study cannot be generalised due to a smaller sample size. The study can be performed with a larger sample size in order to reaffirm the results of this study.

\section{CONCLUSIONS}

Curcumin is a safe, natural herb, giving good results in the healing of the extraction socket in diabetic patients, with definite improvement in the soft tissue healing. The use of Curcuma oral gel can be made as part of a standard set of protocols for post-extraction procedures, especially in cases where there might be a delay in wound healing.

\section{REFERENCES}

[1] Huang S, Dang H, Huynh W, et al. The healing of dental extraction sockets in patients with Type 2 diabetes on oral hypoglycaemics: a prospective cohort. Aust Dent J 2013;58(1):89-93.

[2] Zhang S, Wang S, Wei $\mathrm{H}$, et al. Post-extraction socket changes in diabetic patients - a retrospective study. Int J Clin Exp Med 2016;9(7):14077-86.

[3] Fernandes KS, Kokron CM, Glick M, et al. Post extraction wound healing in patients with type 2 diabetes. Oral Surg Oral Med Oral Pathol Oral Radiol 2013;116(3):e197-e8.

[4] Gupta B. Assessment of post-operative wound healing in diabetic patients after extraction. International Journal of Advances in Scientific Research 2017;3(7):77-81.

[5] Syrjänen SM Syrjänen KJ. Influence of Alvogyl on the healing of extraction wound in man. Int J Oral Surg 1979;8(1):22-30.

[6] Khan A, Khan MA, Nasir A. Comparision of healing of the extraction socket in diabetic vs non-diabetic patients. Journal of Karachi College of Dentistry 2017;7(3):22-6.

[7] Amler MH, Johnson PL, Salman I. Histological and histochemical investigation of human alveolar socket healing in undisturbed extraction wounds. J Am Dent Assoc 1960;61(7):32-44. 
[8] Devlin H, Sloan P. Early bone healing events in the human extraction socket. Int $\mathrm{J}$ Oral Maxillofac Surg 2002;31(6):641-5.

[9] Jacob JN, Badyal DK, Bala S, et al. Evaluation of the in vivo anti-inflammatory and analgesic and in vitro anti-cancer activities of curcumin and its derivatives. Nat Prod Commun 2013;8(3):359-62.

[10] Joe B, Vijaykumar M, Lokesh BR. Biological properties of curcumin-cellular and molecular mechanisms of action. Crit Rev Food Sci Nutr 2004;44(2):97-111.

[11] Panchatcharam M, Miriyala S, Gayathri VS, et al. Curcumin improves wound healing by modulating collagen and decreasing reactive oxygen species. Mol Cell Biochem 2006;290(1-2):87-96.

[12] Akbik D, Ghadiri M, Chrzanowski W, et al. Curcumin as a wound healing agent. Life Sci 2014;116(1):1-7.

[13] Landry RG, Turnbull RS, Howley T. Effectiveness of benzydamyne $\mathrm{HCl}$ in the treatment of periodontal postsurgical patients. Research in Clinic Forums 1988;10:10518.

[14] Nimma VL, Talla HV, Bairi JK, et al. Holistic healing through herbs: effectiveness of Aloe Vera on post extraction socket healing. Journal of Clinical and Diagnostic Research 2017;11(3): ZC83-ZC6.

[15] Lone PA, Ahmed SW, Prasad V, et al. Role of turmeric in management of alveolar osteitis (dry socket): a randomised clinical study. J Oral Biol Craniofac Res 2018;8(1):44-7.

[16] Das AD, Balan A, Sreelatha KT. Comparative study of the efficacy of curcumin and turmeric oil as chemopreventive agents in oral submucous fibrosis: a clinical and histopathological evaluation. J Indian Acad of Oral Med Radiol 2010;22(2):88-92.

[17] Mozzati M, Gallesio G, di Romana S, et al. Efficacy of plasma-rich growth factor in the healing of post extraction sockets in patients affected by insulindependent diabetes mellitus. J Oral Maxillofac Surg 2014;72(3):456-62.

[18] Priya BM, ShivaKumar V, Anitha V, et al. Diabetes \& dental diseases. Chettinad Health City Medical Journal 2012;1(4):188-91.

[19] Kolluru GK, Bir SC, Kevil CG. Endothelial dysfunction and diabetes: effects on angiogenesis, vascular remodeling and wound healing. Article ID 918267, International Journal of Vascular Medicine 2012;2012:1-30.

[20] Herold KC, Huen A, Gould L, et al. Alterations in lymphocyte subpopulations in type 1 (insulin-dependent) diabetes mellitus: exploration of possible mechanisms and relationships to autoimmune phenomena. Diabetologia 1984;(Suppl 27):102-5.

[21] Devlin H, Garland H, Sloan P. Healing of tooth extraction sockets in experimental diabetes mellitus. J Oral Maxillofac Surg 1996;54(9):1087-91.

[22] Mohanty C, Sahoo SK. Curcumin and its topical formulations for wound healing applications. Drug Discov Today 2017;22(10):1582-92.

[23] Mitic A, Todorovic K, Stojiljkovic N, et al. Beneficial effects of curcumin on the wound-healing process after tooth extraction. Nat Prod Commun 2017;12(12):1905-8.

[24] Gadekar R, Saurabh MK, Thakur GS, et al. Study of formulation, characterisation and wound healing potential of transdermal patches of curcumin. Asian J Pharm Clin Res 2012;5(Suppl 4):225-30.

[25] Maulina T, Diana H, Cahyanto A, et al. The efficacy of curcumin in managing acute inflammation pain on the post-surgical removal of impacted third molars patients: a randomised controlled trial. J Oral Rehabil 2018;45(9):677-83.

[26] Jurenka JS. Anti-inflammatory properties of curcumin, a major constituent of curcumin longa: a review of preclinical and clinical research. Altern Med Rev 2009;14(2):141-53.

[27] Chang WK, Wu HL, Yang CS, et al. Effect on pain relief and inflammatory response following addition of tenoxicam to intravenous patient-controlled morphine analgesia: a double-blind, randomized, controlled study in patients undergoing spine fusion surgery. Pain Med 2013;14(5):736-48.

[28] Goel A, Kunnumakkara AB, Anggarwal BB. Curcumin as "curecumin": from kitchen to clinic. Biochem Pharmacol 2008;75(4):787-809.

[29] Abe Y, Hashimoto S, Horie T. Curcumin inhibition of inflammatory cytokine production by human peripheral blood monocytes and alveolar macrophages. Pharmacol Res 1999;39(1):41-7. 